\title{
Asymmetric Y-shaped Micromixers with Spherical Mixing Chamber for Enhanced Mixing Efficiency and Reduced Flow Impedance
}

\author{
E. Cetkin ${ }^{1}$ and A. F. Miguel ${ }^{2,3 \dagger}$ \\ ${ }^{1}$ Department of Mechanical Engineering, Izmir Institute of Technology, Turkey \\ ${ }^{2}$ Department of Physics, School of Science and Technology, University of Evora, Portugal \\ ${ }^{3}$ Institute of Earth Sciences (ICT), Pole of Evora, 7000-671, Evora, Portugal
}

†Corresponding Author Email: afm@ uevora.pt

(Received October 25, 2020; accepted February 11, 2021)

\begin{abstract}
Microfluidic devices have many attractive qualities such as low cost, small size, and in-field use. Micromixers are very important components of these devices because affect their efficiency. In a passive mixer, the structural characteristics of the mixer are crucial and must be analyzed. This paper presents a numerical study of the mixing in passive Y-shaped micromixers with a spherical mixing chamber for a volume constrained system. The effect of asymmetric bifurcated ducts, the angle in between the inflow ducts, eccentricity and, obstacles inserted in the mixing sphere, on the mixing efficiency and flow impedance is evaluated. Vortical structures characteristics and the possible occurrence of engulfment are also identified. The results show that flow impedance (pressure drop for unit volumetric flow rate) can be decreased greatly for the same mixing efficiency as the volume of the spherical mixing chamber is $20 \%$ of the total volume. Insertion of the obstacles into the sphere mixing chamber decreases the mixing efficiency while they increase the flow impedance. The results also show that spherical mixing chamber enhances mixing efficiency while decreasing flow impedance if the volume reserved for it is greater than a limit value which depends on the diameter and length scale ratios in between the mother and daughter ducts as well as the total volume. Overall, the paper documents the variation of mixing efficiency and flow impedance based on the geometrical parameters of three-dimensional asymmetric passive micromixer with sphere mixing chamber.
\end{abstract}

Keywords: Micromixer; Asymmetric Y-mixer; Spherical mixing chamber; Obstacles.

\section{NOMENCLATURE}

$\begin{array}{ll}\text { A } & \text { surface area } \\ \text { c } & \text { concentration } \\ \text { D } & \text { channel diameter } \\ \mathrm{D}_{\mathrm{c}} & \text { diffusion coefficient } \\ \mathrm{L} & \text { channel length } \\ \mathrm{M} & \text { mixing efficiency } \\ \mathrm{P} & \text { pressure } \\ \mathrm{Q} & \text { volumetric flow rate } \\ \mathbf{u} & \text { velocity vector } \\ \mathrm{U} & \text { velocity magnitude } \\ \mathrm{V} & \text { volume } \\ \mathrm{Xecc}_{\text {ecc }} & \text { eccentricity }\end{array}$

\section{INTRODUCTION}

Microfluidic technology has received great attention due to its importance in many diverse fields, such as

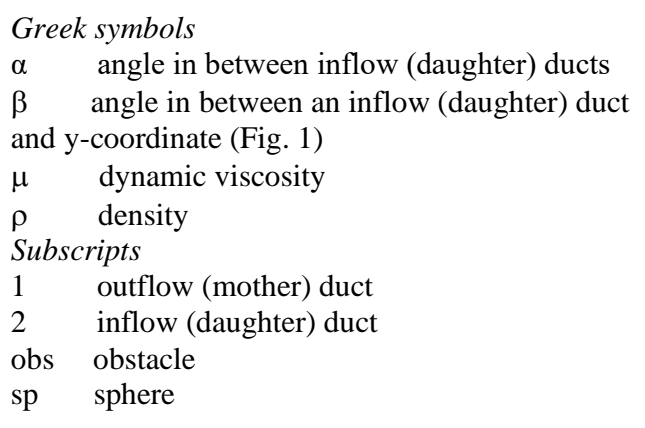

chemistry, medicine, biology and physical sciences (Tabeling 2005; Nguyen and Wu 2005; Capretto et al. 2011; Ababaei et al. 2017; Lee and Fu 2018; Saleel et al. 2020). This technology has several 
advantages that includes the use of small amounts of samples and reagents, high accuracy and reliability, low cost and short processing time and high portability. An essential component of this technology is the micromixer that together with components are on the basis of lab-on-a-chip devices. Micromixers can be classified as belonging to one of the two categories: active and passive. The main difference between them is that the first relies on external energy sources, while the second relies on geometrical characteristics. Comprehensive reviews on micromixers are presented elsewhere (e.g., Nguyen and Wu 2005; Capretto et al. 2011; Ababaei et al. 2017; Lee and Fu 2018; Cetkin and Miguel 2019; Saleel et al. 2020).

This paper is about passive mixers which are robust, easy integration and simple manufacturing. T- and Y-shaped micromixers, in which the inlet of two streams are contacted at the junction where the mixing process begins, are basic components of any complex micromixing apparatus (Tabeling 2005; Lee and Fu 2018). At very low Reynolds number, fluid flow is laminar, and mixing is due to diffusion. Increasing the Reynolds number becomes a steady symmetric vortex flow, and then a steady asymmetric vortex flow (i.e., an engulfment regime because of the similarity to the structures originated in turbulent flow). The transition from symmetric to asymmetric flow, which is generated even in symmetric configurations (Fani et al. 2013; Cetkin and Miguel 2019), increases mixing efficiency. The critical Reynolds number for the transition to asymmetric flow depends on the geometry of the bifurcated geometry (e.g., size, asymmetry of Tand Y-shaped channels (Fani et al. 2013; Cetkin and Miguel 2019)), and thermophysical properties of mixing fluids (Orsi et al. 2013; Lobasov and Minakov 2018; Shah et al. 2020). Despite the benefit of operating above the critical Reynolds number on enhanced mixing efficiency, there is an increase of power input to operate the device. Other possible solution relies on altering the design of passive mixers to enhance mixing efficiency. Attempts are made by either varying the shape of the channels by inserting obstacles, ridges, baffles, grooves, curves in the flow paths (Kim et al. 2011; Cook et al. 2013; Alam et al. 2014; Kang 2015; Marschewski et al. 2016; Ritter et al. 2016; Li and Chen 2017; Chen and Zhao 2017; Wangikar et al. 2018; Cetkin and Miguel 2019; Zhan et al. 2020) and by split-recombine chaotic design of micromixers (Viktorov et al. 2016, Chen and Shen 2017; Hermann et al. 2018; Shaha et al. 2019). Furthermore, (Madadelahi et al. 2020) reviews the theoretical aspects and numerical studies in the field of centrifugal microfluidic devices which includes the essential equations and fundamental concepts in microfluidics.

The above modifications in the mixer geometry may increase the surface area and disturb the fluid streams which yield an increase in mixing performance but also lead to pressure drop (flow impedance) penalties. So, there is a fundamental tradeoff between the two trends and the chief result to obtain should be the best design to provide great mixing and access to streams. For the best design to emerge, size constraints must be included in the analysis. The emergence of designs that offer progressively greater access to streams to achieve that goal, under constraints, can be obtained in light of a principle - the constructal law (Bejan 2000, 2008; Bejan and Lorente 2008; Miguel 2010) This law of configuration generation was used to predict basic feature of natural and engineered flow systems (see, e.g., (Bejan 2000, 2012; Miguel 2013, 2016, 2019; Cetkin 2017; Lucia and Deisboeck 2018; Soni et al. 2020).

In the present paper, mixing process in $\mathrm{Y}$-mixer with spherical mixing chamber is studied and characterized by means of numerical simulation. We focus herein on the identification of the effect of asymmetric ducts, angle in between the inflow ducts, eccentricity and obstacles inserted in the mixing sphere, on the mixing efficiency and flow impedance is analyzed. Unlike the current literature, this paper documents the effect of geometrical parameters of a 3-D micromixer such as angles in between mother and daughter ducts to increase residency time in the spherical mixing chamber. This approach yields enhancement in the mixing efficiency while uncovering how the pressure drop can be minimized.

\section{MODEL}

We consider here a Y-shaped mixer made by two inflow ducts (i.e., daughter ducts) connected with a spherical mixing chamber that is attached to an outflow duct (i.e., mother duct), depicted in Fig. 1. The cross sections of the ducts are circular. Diameter and length of the mother and daughter ducts are $D_{1}$ and $L_{1}$, and $D_{2}$ and $L_{2}$, respectively, $\propto$ is the angle in between the daughter ducts, and $\beta$ is the angle in between one of the daughter ducts and $\mathrm{z}$-coordinate as shown in Fig. 1. The other daughter duct does not rotate along the $\mathrm{z}$-axis $(\beta=0)$ which creates asymmetry even the length and diameter of the daughter ducts are the same. To take into account the fact that space is limited, the volume of the flow domain ( 2 daughter ducts, 1 mother duct and a mixing chamber) is constrained to a fixed value $\left(V=10^{-10} \mathrm{~m}^{3}\right)$. The concentrations (c) at the inlet ducts are 1 and 0 , where the species are carried with water at the room temperature with the density of $\rho=10^{3} \mathrm{~kg} / \mathrm{m}^{3}$ and dynamic viscosity of $\mu=10^{-3}$ Pa.s . In addition, the diffusion coefficient is $D_{c}=10^{-9} \mathrm{~m}^{2} / \mathrm{s}$. The fluids enter to daughter ducts at fixed Reynolds number (i.e., inlet velocity varies as diameters change but Re is 100). There is no mixing along the inlet ducts, and fluids begin to interact at the spherical mixing chamber (Fig. 1). Because the inlet of daughter ducts is asymmetric, the momentum of the fluids entering to the mixing chamber from the daughter ducts yield additional rotation to the fluid inside the mixing chamber. Therefore, the time spent in the mixing chamber and interaction between the low and high concentration fluid packages increases. The mixing 
of the fluids continues until they leave from the outlet surface of the mother duct.

(a)
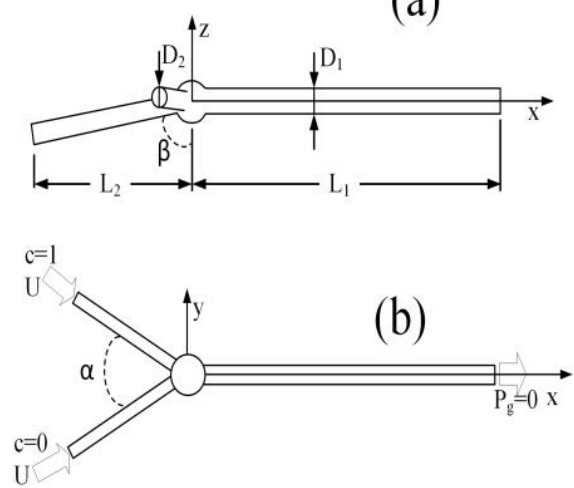

Fig. 1. (a) Design of 3-D asymmetric Y-shaped micromixer with spherical mixing chamber and (b) top view of it with boundary conditions.

Incompressible flows of multi-component can be described as Newtonian fluids since the ducts size are greater than $1 \mu \mathrm{m}$ (Omori et al. 2012; Miguel 2015). We consider here the incompressible, laminar and steady state flow inside the mixer (Fig. 1), where fluid is single phase with constant material properties. The fluid motion is described by the three-dimensional incompressible NavierStokes equations.

$\nabla \cdot u=0$

$\rho(u . \nabla) u=-\nabla P+\mu \nabla^{2} u$

where $\mathrm{u}$ is the velocity vector, $\mathrm{P}$ is the pressure, $\mu$ is the dynamic viscosity, and $\rho$ is the density.

The velocity field by solving the conservation of mass and momentum equations (Eqs. 1-2) was inserted into the convection-diffusion equation, Eq. 3 , which governs the distribution of the concentration.

$u \cdot \nabla c=D_{c} \nabla^{2} c$

where $\mathrm{c}$ is the concentration, and $\mathrm{D}_{\mathrm{c}}$ is the diffusion coefficient.

No-slip boundary conditions were imposed at the mixer walls, while a unidirectional fully developed velocity profile is assumed at the inlet ports. Outflow condition is zero gage pressure.

The mixing efficiency is defined as (Cetkin and Miguel 2019)

$M=1-\sqrt{\frac{\iint\left(\frac{c_{i}-\bar{c}}{\bar{c}}\right)^{2} d A}{\iint d A}}$

Where $\mathrm{c}_{\mathrm{i}}$ and $\bar{c}$ are local and average concentrations at the surface of interest, respectively, and $\mathrm{dA}$ denotes derivation of the surface area through which mixture flows. Here $\left(\frac{c_{i}-\bar{c}}{\bar{c}}\right)^{2}$ is integrated over the surface of interest (outlet port of the mother duct), and then it is divided to the surface area of it.

The governing equations were solved relative to the boundary conditions as shown in Fig. 1 by using a finite element software, COMSOL Multiphysics 5.0 (Comsol, 2014). The solution procedure is the same as described in Cetkin and Miguel (2019), where unstructured mesh elements with 5 layers of boundary layer meshes were applied, and the convergence criterion during the simulations was $10^{-6}$. 463725 number of mesh elements conform mesh independency (less than $1 \%$ relative error), and the current study was also validated based on the work of (Chen and Zhao 2017) where the details can be found in (Cetkin and Miguel 2019).

\section{ASYMMETRIC Y-SHAPED MIXER WITH A SPHERICAL MIXING CHAMBER}

Consider the asymmetric mixer with a spherical mixing chamber between two inflows and one outflow ducts (Fig. 1). Size-limiting constraints are fixed volume of the mixer $\left(V=10^{-10} \mathrm{~m}^{3}\right)$, and fixed ratio of the spherical mixing chamber volume relative to the entire volume of the mixer $\left(\mathrm{V}_{\mathrm{sp}}=0.1\right)$. The effect of eccentricity ( $\mathrm{x}_{\mathrm{ecc}}$ ) of the imaginary intersection point of the daughter ducts from the origin of the sphere is surveyed parametrically.

Figure 2 shows the effect of both the angle $\propto$ and the eccentricity on mixing efficiency (Eq. 4) and flow impedance (pressure drop over volumetric flow rate, $\Delta \mathrm{P} / \mathrm{Q}$ ) when $\beta=0^{\circ}$ (i.e., the angle in between the inflow (daughter) ducts and z-coordinate is zero (Fig. 1)). Both mixing efficiency and flow impedance increases as $\propto$ increases. However, for a given $\propto$, eccentricity may increase or decrease mixing efficiency and flow impedance for the considered geometrical length scale ratios. Notice that the maximum increase in the mixing efficiency and flow impedance are 2 and $1.5 \%$, respectively. In general, the results show that the eccentricity slightly affect the mixing efficiency. An eccentricity of $30 \mu \mathrm{m}$ which corresponds to the optimal mixing efficiency and flow impedance will be considered for the mixing chamber designs afterwards.

Figure 3 documents how $\alpha$ and $\beta$ angles, can be seen in Fig. 1, affect the mixing efficiency (Eq. 4) and the flow impedance $(\Delta \mathrm{P} / \mathrm{Q})$ for $\mathrm{L}_{1} / \mathrm{L}_{2}=6$, $\mathrm{L}_{2} / \mathrm{D}_{2}=10$ and $\mathrm{D}_{1} / \mathrm{D}_{2}=1$. Figure $3 \mathrm{a}$ shows that increasing $\beta$ from $0^{\circ}$ to $10^{\circ}$ increases the mixing efficiency. This is a remarkable result that documents that the design of the micromixer should be three-dimensional in order to maximize mixing efficiency. Figure $3 \mathrm{a}$ also shows that, for the angles between inlet ducts greater than $40^{\circ}$, the mixing efficiency decreases as $\beta$ increases from 10 to $40-60^{\circ}$. Then increasing $\beta$ yields a fluctuating behavior on the mixing efficiency. 

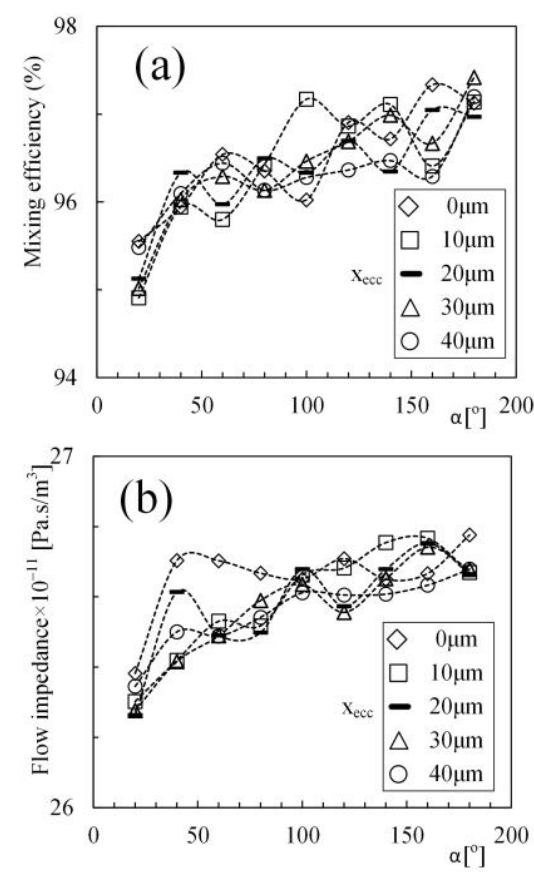

Fig. 2. Effect of eccentricity of the origins $\left(x_{\text {ecc }}\right)$ and $\propto$ on (a) the mixing efficiency and (b) flow impedance for $L_{1} / L_{2}=6, L_{2} / D_{2}=10$ and $D_{1} / D_{2}=1$.
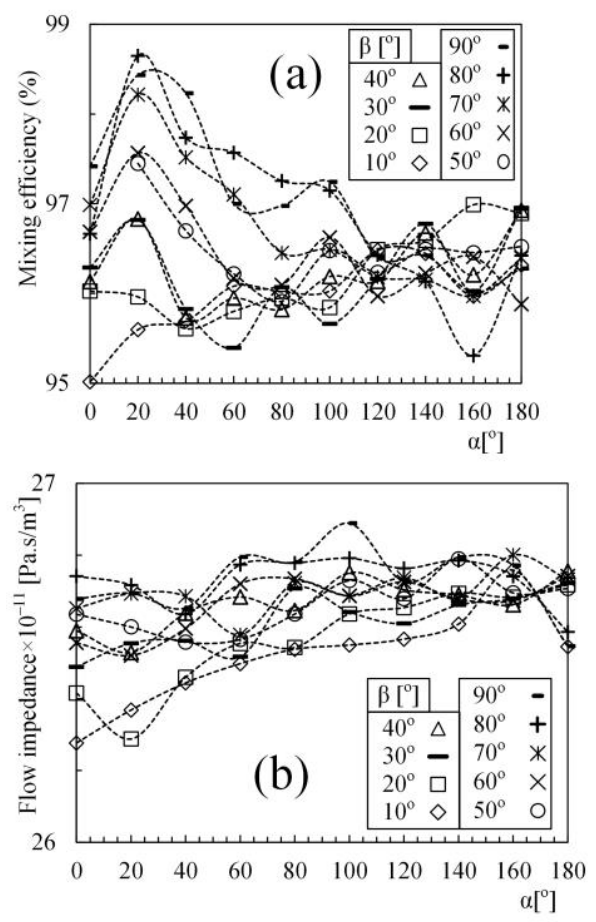

Fig. 3. Effect of $\alpha$ and $\beta$ on (a) the mixing efficiency and (b) impedance for $L_{1} / L_{2}=6$, $L_{2} / D_{2}=10, D_{1} / D_{2}=1$, and $x_{e c c}=30 \mu \mathrm{m}$.

In addition, this plot shows that for $\alpha$ lower than $40^{\circ}$, the mixing efficiency fluctuates as $\beta$ increases. However, it should be noted that the maximum difference is about 3\% (Fig. 3a). In summary, fluids mix more efficiently in the spherical chamber if $\beta>0$, because fluids rotate, and the interaction time increases.
The effect of $\alpha$ and $\beta$ on the flow impedance is depicted at Fig. 3b. Notice that the flow impedance variation is very small, less than $2 \%$.

Next, the angles $\alpha$ and $\beta$ corresponding to the maximum mixing efficiency are fixed, and the effect of size ratios of successive ducts segments $L_{1} / L_{2}$ and $D_{1} / D_{2}$ were analyzed. Figure $4 a$ shows that the mixing efficiency increases as $\mathrm{L}_{1} / \mathrm{L}_{2}$ increases. This means that the outlet duct not only conducts fluids but plays an integral role in mixing process.
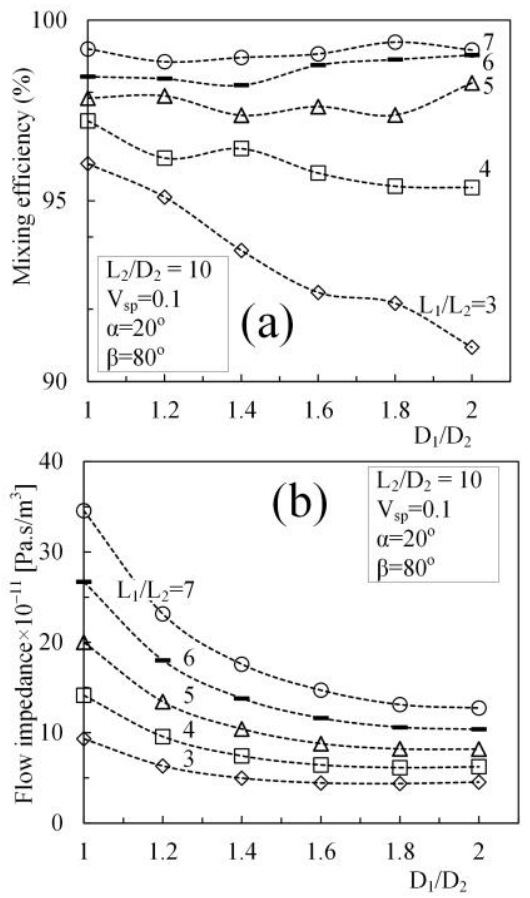

Fig. 4. Effect of $L_{1} / L_{2}$ and $D_{1} / D_{2}$ on (a) the mixing efficiency and (b) impedance when $\alpha$ and $\beta$ are 20 and $80^{\circ}$ with $L_{2} / D_{2}=10$ and $x_{e c c}=30 \mu \mathrm{m}$.

The effect of $D_{1} / D_{2}$ on the mixing efficiency is also shown in Fig. 4a. This figure shows that increasing $\mathrm{D}_{1} / \mathrm{D}_{2}$ does not necessarily mean an increase in mixing efficiency, as does a simple Y-mixer without mixing chamber (Cetkin and Miguel 2019). For $\mathrm{L}_{1} / \mathrm{L}_{2}<5$, the mixing efficiency decreases with $\mathrm{D}_{1} / \mathrm{D}_{2}$, but for length ratios equal to or greater than 5 , the mixing efficiency is almost constant. Please note that $\mathrm{V}_{\mathrm{sp}}$ is 0.1 . Therefore, for low $\mathrm{L}_{1} / \mathrm{L}_{2}$ ratios diameter of the sphere mixing chamber is smaller than the sudden diameter changes in between the daughter and mother channels. This also decreases the volume which would be occupied by the mother and daughter channels, i.e. decreased length. These factors decrease in the mixing efficiency when $\mathrm{L}_{1} / \mathrm{L}_{2}<5$ as the dominant mixing is occurring along the mother channel due to diffusion. Therefore, this transition limit shows when the mixing efficiency becomes more with sphere mixing chamber than without it, i.e. mixing dominated by diffusion along the mother channel. Note that this transition limit would change as $V_{s p}$ varies, i.e. as $V_{s p}$ decreases its effect on the mixing is expected to diminish. As presumed, decreasing $\mathrm{L}_{1} / \mathrm{L}_{2}$ and increasing $\mathrm{D}_{1} / \mathrm{D}_{2}$ 
ratios decrease the flow impedance (Fig. 4b). Notice that a flow system with $\mathrm{L}_{1} / \mathrm{L}_{2}=5$ represents a good trade-off between mixing efficiency and flow impedance. It is also remarkable to find that the $\mathrm{Y}$ mixer with mixing chamber (Fig. 1), with optimized eccentricity and $\beta$ enhances mixing efficiency and decreases flow impedance compared to a simple $\mathrm{Y}$ mixer (see Fig. 13 of Cetkin and Miguel (2019). For $\mathrm{L}_{1} / \mathrm{L}_{2}=4$ and $\mathrm{D}_{1} / \mathrm{D}_{2}=1$, there is an increase of $6 \%$ in mixing efficiency and a decrease of more than $50 \%$ in the flow impedance.

We shall now consider that $\mathrm{L}_{1} / \mathrm{L}_{2}$ is fixed as 3 . The effect of spherical mixing chamber volume fraction and $\mathrm{D}_{1} / \mathrm{D}_{2}$ ratio on both the mixing efficiency and flow impedance is depicted at Fig. 5. According to this plot, increasing the volume of the spherical mixing chamber increases both mixing efficiency and flow impedance. It is important to observe that for $\mathrm{V}_{\mathrm{sp}}=0.25$, the mixing efficiency is practically independent of $\mathrm{D}_{1} / \mathrm{D}_{2}$, and higher than $95 \%$ (Fig. 5a). Furthermore, the flow impedance can be minimized for $\mathrm{D}_{1} / \mathrm{D}_{2}=1.8$ (Fig. $5 \mathrm{~b}$ ). Note that flow impedance varies less than $3 \%$ when $\mathrm{D}_{1} / \mathrm{D}_{2}$ varies from 1.6 to 2 . In addition, Fig. 5 confirms the reasoning deduced from the results of Fig. 4 because increase in the $V_{s p}$ enhances the mixing efficiency comparatively more if $D_{1} / D_{2}$ ratio is greater.
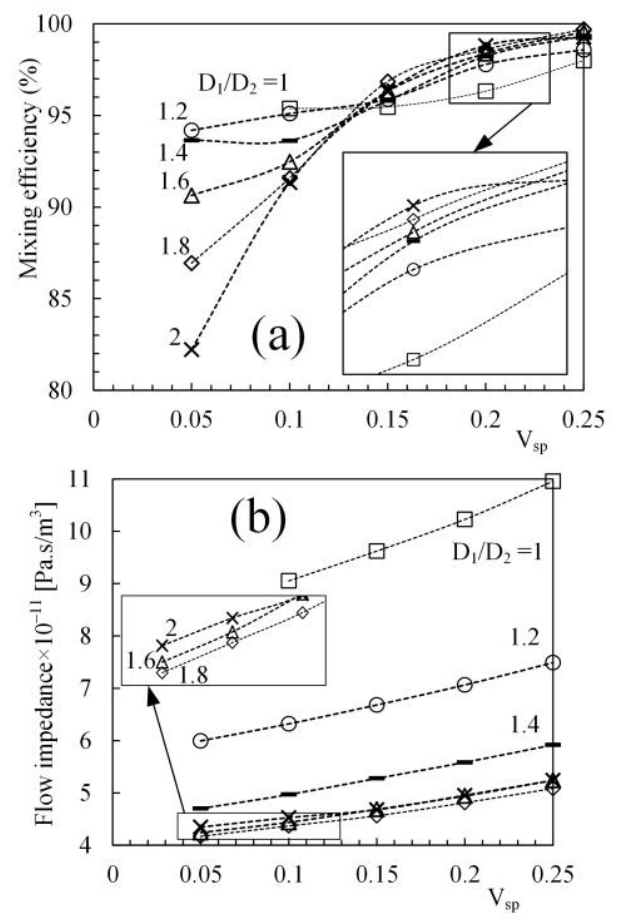

Fig. 5. Effect of $V_{\text {sp }}$ and $D_{1} / D_{2}$ on (a) the mixing efficiency and (b) impedance for the design of Fig. 4 with $L_{1} / L_{2}=3$.

As expected, flow impedance increases as $V_{\mathrm{sp}}$ increases too. However, Figs. $4 \mathrm{~b}$ and $5 \mathrm{~b}$ shows that the effect of $V_{s p}$ on flow impedance is relatively small in comparison to the effect of $\mathrm{L}_{1} / \mathrm{L}_{2}$. These results clearly indicate that for $\mathrm{V}_{\mathrm{sp}} \sim 0.2, \mathrm{D}_{1} / \mathrm{D}_{2} \sim 1.6$ and $\mathrm{L}_{1} / \mathrm{L}_{2} \sim 3$ the mixing efficiency is greater than
95\% and the value of flow impedance is relatively low in comparison to the competing designs.

Figure 6 shows the velocity streamlines versus the concentration field, where blue and red colors represent 0 and 1 concentrations, respectively. Figure 6a shows that the mixing occurs almost entirely in the mixing chamber when $\mathrm{D}_{1} / \mathrm{D}_{2}=1.8$, $\mathrm{V}_{\mathrm{sp}}=0.25$ and, $\mathrm{L}_{1} / \mathrm{L}_{2}=3$. To understand how the fluids are mixing within the spherical chamber, the section near the mixing chamber is depicted at Fig. 6b. This figure shows that fluids with different concentrations collide near the origin of the sphere and rotate in the mixing chamber before entering to the mother channel. Notice that at spherical chamber with volume $\mathrm{V}_{\mathrm{sp}}=0.05$ (Fig. 6c), fluids collide but does not rotate before they flow to the outflow duct. Therefore, most of the mixing occur along the outflow duct. This explains why the mixing efficiency increases as the mixing chamber volume increases too. Overall, concentration streamlines also confirm the existence of a limit value for the spherical mixing chamber to enhance mixing efficiency as deduced from the results depicted in Figs. 4 and 5.

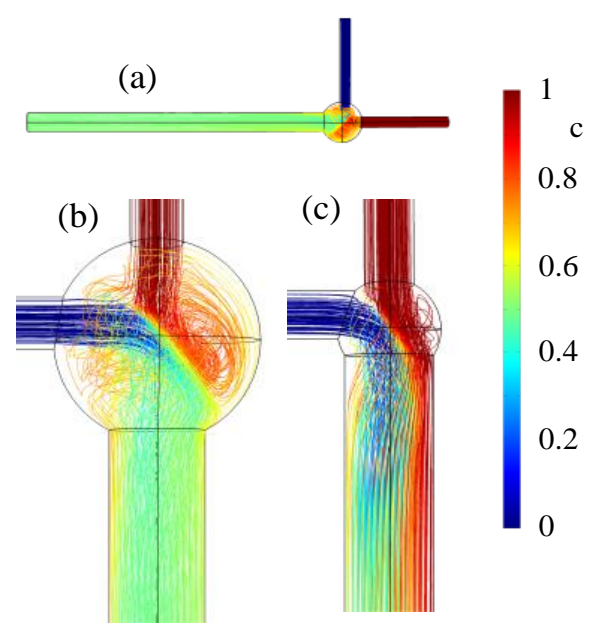

Fig. 6. Velocity streamlines for the designs with $D_{1} / D_{2}=1.8$ and $L_{1} / L_{2}=3$, where blue and red colors represent concentrations of 0 and 1 : (a) $\mathrm{V}_{\mathrm{sp}}=\mathbf{0 . 2 5}$, (b) $\mathrm{V}_{\mathrm{sp}}=\mathbf{0 . 2 5}$ (detail of mixing chamber) and (c) $V_{\mathrm{sp}}=\mathbf{0 . 0 5}$ (detail of mixing chamber).

\section{Y-MIXER WITH OBSTACLES INSERTED IN THE SPHERICAL CHAMBER}

Studies show that the obstacles embedded along the outflow duct enhance the mixing because disrupt flow and reduce diffusion path, and therefore its length can be reduced (Nguyen 2005; Cetkin and Miguel 2019). Obstacles may be placed inside the spherical chamber, that connects inflow ducts to outflow duct. Next, we consider that the obstacles are embedded inside the spherical chamber. The ratio between lengths $L_{1} / L_{2}$ is fixed at 2 and 3 , while the obstacles and spherical chamber volumes vary. Obstacles are embedded in between the center 
of spherical chamber and the entrance of mother duct (see Fig. 7a), and their volume does not change the total flow domain volume.

Figures 7 and 8 show the results for micromixers with 7 embedded obstacles. The variation of mixing efficiency and flow impedance with the spherical chamber and various obstacle volume fractions are represented in Figs. $7 \mathrm{~b}$ and $7 \mathrm{c}$. According to these figures, mixing efficiency increases with the spherical chamber volume fraction, but surprisingly, it decreases with the obstacle volume fraction. This means that obstacles placed near the outlet of spherical chamber have a negative impact on mixing (Fig. 8). As expected, both the increasing of obstacles volume ratio and the decreasing of spherical chamber volume ratio increase the flow impedance. In summary, embedding obstacles into the spherical mixing chamber has a double penalty because it does not enhance the mixing efficiency while it increases flow impedance.
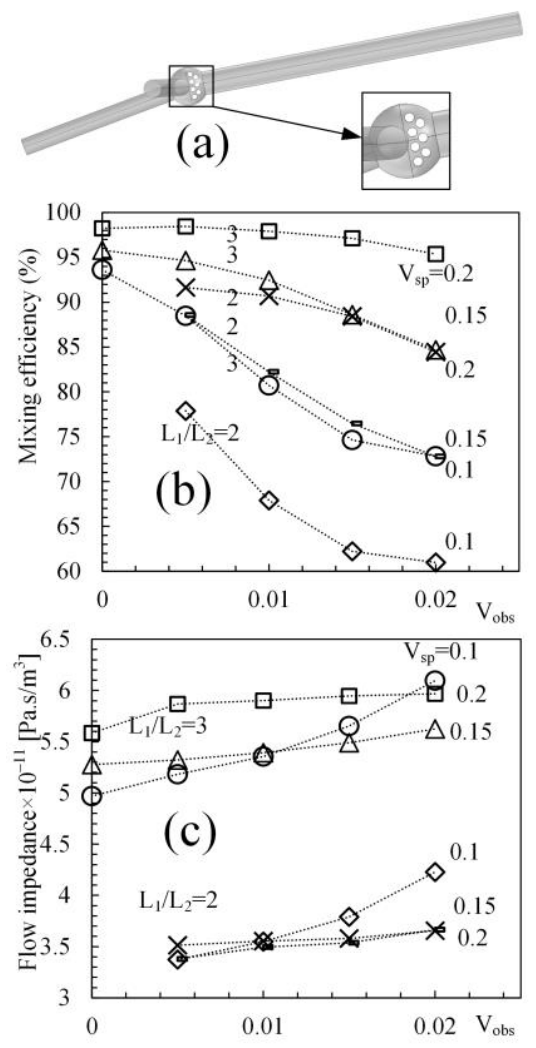

Fig. 7. Y-mixer with 7 embedded obstacles in the spherical chamber $\left(\alpha=20^{\circ}, \beta=\mathbf{8 0}^{\circ}, x_{\mathrm{ecc}}=30 \mu \mathrm{m}\right)$ :

(a) Detail of position of embedded obstacles within the spherical chamber, (b) Mixing efficiency vs. $V_{\text {sp }}$ and $V_{\text {obs, }}$ and (c) Flow impedance vs. $V_{\text {sp }}$ and $V_{\text {obs. }}$

The number of obstacles that are embedded in between the center of spherical chamber and the entrance of the mother duct is increased to 9 for the same length scales of Fig. 7. The results for micromixers with 9 embedded obstacles are depicted at Figs. 9 and 10. In general, the profile of variation of mixing efficiency and flow impedance

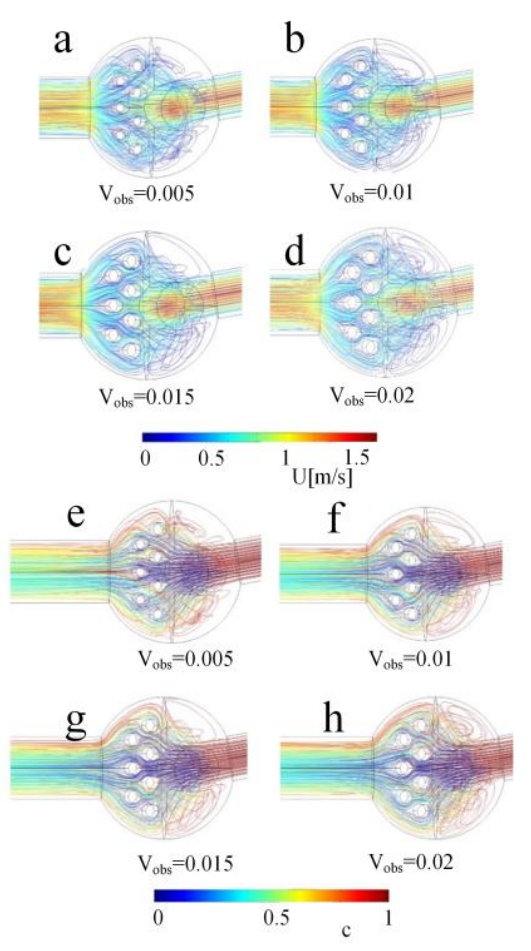

Fig. 8. Velocity (a, b, c and d) and concentration streamlines $\left(e, f, g\right.$ and $h$ ) for $V_{\text {obs }}=0.005,0.01$, 0.015 and 0.02 , respectively, for $Y$-mixer with 7 embedded obstacles in the spherical chamber, $\alpha=20^{\circ}, \beta=80^{\circ}, x_{\text {ecc }}=30 \mu \mathrm{m}, V_{\mathrm{sp}}=0.2, \mathrm{~L}_{1} / \mathrm{L}_{2}=2$.
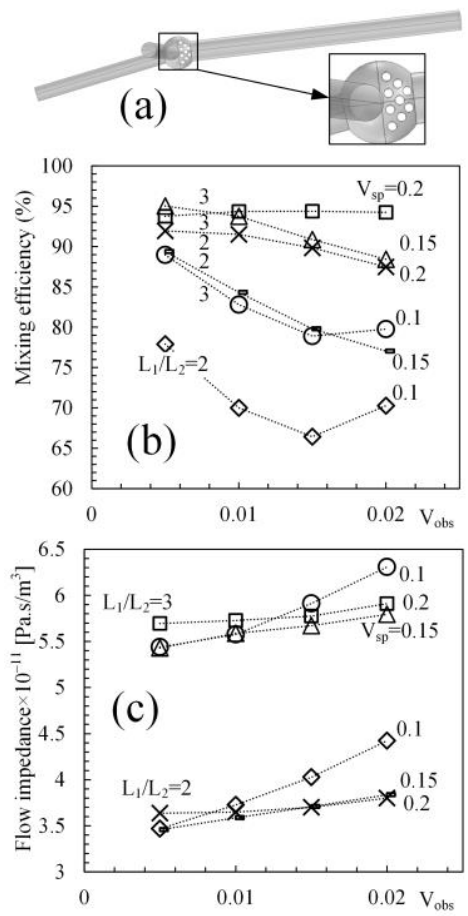

Fig. 9. Y-shaped mixer with 9 embedded obstacles in the spherical chamber $\left(\alpha=20^{\circ}\right.$, $\beta=80^{\circ}, x_{\text {ecc }}=30 \mu \mathrm{m}$ ): (a) Detail of position of embedded obstacles within the spherical chamber, (b) Mixing efficiency vs. $V_{\text {sp }}$ and $V_{\text {obs }}$, and (c) Flow impedance vs. $V_{s p}$ and $V_{\text {obs. }}$. 


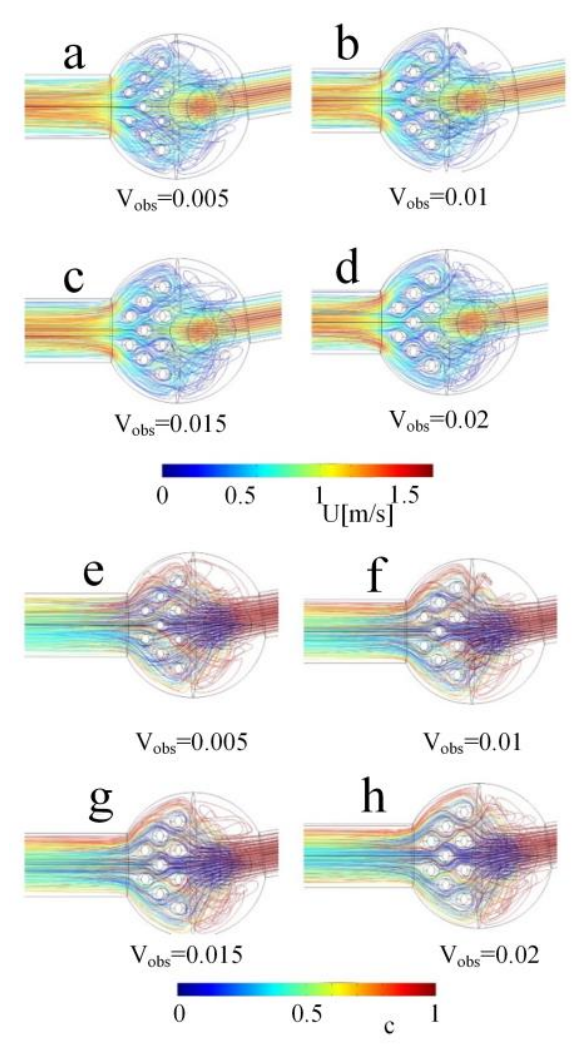

Fig. 10. Velocity (a, b, c and d) and concentration streamlines $\left(e, f, g\right.$ and $h$ ) for $V_{\text {obs }}=0.005,0.01$, 0.015 and 0.02 , respectively, for $Y$-mixer with 9 embedded obstacles in the spherical chamber, $\alpha=20^{\circ}, \beta=80^{\circ}, x_{\text {ecc }}=30 \mu \mathrm{m}, V_{\mathrm{sp}}=0.2, \mathrm{~L}_{1} / \mathrm{L}_{2}=3$.

is similar for Y-mixer with 7 and 9 embedded obstacles. These figures also reveal that the mixing efficiency decreases as the number of obstacles increases from 7 to 9 , except for $\mathrm{V}_{\mathrm{obs}}>0.015$ and $\mathrm{V}_{\mathrm{sp}}=0.1$ (there is an increase of mixing efficiency), and for $\mathrm{V}_{\mathrm{sp}}=0.2$ and $\mathrm{L}_{1} / \mathrm{L}_{2}=3$ (mixing efficiency is constant). In summary, the increase in complexity by introducing obstacles in the mixing sphere is counterproductive. Under similar geometrical and flow conditions, a smooth spherical chamber generates much better mixing efficiencies and flow impedances than an obstacle-embedded spherical chamber for similar geometrical and flow conditions.

\section{Conclusions}

Passive micromixer that consists of two asymmetric inflow ducts and an outflow duct linked by a mixing sphere in between is studied numerically. Two designs were considered for volume constrained systems: mixing sphere with and without obstacles inserted in it. The performance was evaluated for various outflow to inflows duct size ratios, angles between inflow ducts, angle in between an inflow duct and z-coordinate, and eccentricity. The results allow us to draw several conclusions.

Micromixer design must be considered always as three-dimensional. Only then parameters such as the eccentricity can be included. Asymmetric inflow ducts improve the mixing efficiency. Length and diameter ratios between outflow and inflows ducts influence both the mixing efficiency and the flow impedance. The mixing efficiency increases as the length ratio of outflow duct to inflow ducts increases too. In general, mixing efficiency and flow impedance increase with both the angle between inflow ducts, and the angle in between an inflow duct and z-coordinate. However, the eccentricity may increase or decrease the mixing efficiency and flow impedance. The results also show that mixing efficiency increases with the insertion of spherical mixing chamber if the volume allocated for the mixing chamber is greater than a limit value which depends on the ratio of the mother and daughter channel diameters and overall volume. Mixing efficiency decreases if allocated volume is less than this limit value. Therefore, embedding spherical mixing chamber does not automatically ensure enhancement in the mixing efficiency. Finally, introducing obstacles in the mixing sphere decrease the mixing efficiency, which is lower with a smaller number of obstacles. A smooth spherical chamber provides a better performance because flows are freer to rotate and the interaction time in the mixing chamber is greater.

\section{REFERENCES}

Ababaei, A., A. A. Arani and A. Aghaei (2017). Numerical investigation of forced convection of nanofluid flow in microchannels: effect of adding micromixer. Journal of Applied Fluid Mechanics 10, 1759-1772.

Ahamed Saleel, C., A. Algahtani, I. Anjum Badruddin, T. M. Yunus Khan, S. Kamangar and M. A. H. Abdelmohimen (2020) Pressure-driven electro-osmotic flow and mass transport in constricted mixing microchannels. Journal of Applied Fluid Mechanics $13,429-441$.

Alam, A., A. Afzal and K. Y. Kim (2014) Mixing performance of a planar micromixer with circular obstructions in a curved microchannel. Chem. Eng. Res. Design 92, 423-434.

Bejan, A. (2000) Shape and Structure, from Engineering to Nature. Cambridge University Press, Cambridge.

Bejan, A. (2012) Why the bigger live longer and travel farther: animals, vehicles, rivers and the winds. Scientific Reports 2, 594.

Bejan, A. and S. Lorente (2008) Design with Constructal Theory. Wiley, Hoboken.

Capretto, L., W. Cheng, M. Hill and X. Zhang (2011) Micromixing within microfluidic devices. Top. Curr. Chem 304, 27-68.

Cetkin, E. (2017) Constructal microdevice manifold design with uniform flow rate distribution by consideration of the tree branching rule of Leonardo da Vinci and Hess-Murray rule. Journal of Heat Transfer 139, 082401-1- 
082401-9.

Cetkin, E. and A.F. Miguel (2019) Constructal branched micromixers with enhanced mixing efficiency: slender design, sphere mixing chamber and obstacles. International Journal of Heat and Mass Transfer 131, 633-644.

Chen, X. and J. Shen (2017) Numerical analysis of mixing behaviors of two types of $\mathrm{E}$ shape micromixers. International Journal of Heat Mass Transfer 106, 593-600.

Chen, X. and Z. Zhao (2017) Numerical investigation on layout optimization of obstacles in a three-dimensional passive micromixer. Analytica Chimica Acta 964, 142-149.

COMSOL Multiphysics 5.0 (2014) COMSOL Inc., https://www.comsol.com/.

Cook, K. J., Y. Fan and I. Hassan (2013) Mixing evaluation of a passive scaled-up serpentine micromixer with slanted grooves. Journal of Fluids Engineering 135, 081102.

Fani, A., S. Camarri and M. V. Salvetti (2013) Investigation of the steady engulfment regime in a three-dimensional T-mixer. Physics of Fluids 25, 064102.

Hermann, P., J. Timmermann, M. Hoffmann, M. Schlüter, C. Hofmann, P. Löb and D. Ziegenbalg (2018) Optimization of a split and recombine micromixer by improved exploitation of secondary flows. Chemical Engineering Journal 334, 1996-2003.

Kang, D. (2015) Effects of baffle configuration on mixing in a T-shaped micro-channel. Micromachines 6, 765-777.

Kim, B. S., B. S. Kwak, S. Shin, S., Lee, K. M. Kim, H. I. Jung and H. H. Cho (2011) Optimization of microscale vortex generators in a microchannel using advanced response surface method. International Journal of Heat Mass Transfer 54, 118-125.

Lee, C. Y. and L. M. Fu (2018) Recent advances and applications of micromixers. Sensors \& Actuators B 259, 677-702.

Li, T. and X. Chen (2017) Numerical investigation of 3D novel chaotic micromixers with obstacles. International Journal of Heat and Mass Transfer 115, 278-282.

Lobasov, A. S. and A. V. Minakov (2018) Analyzing mixing quality in a T-shaped micromixer for different fluids properties through numerical simulation. Chemical Engineering Process. Intensif 124, 11-23.

Lucia, U. and T. S. Deisboeck (2018) The importance of ion fluxes for cancer proliferation and metastasis: a thermodynamic analysis. Journal of Theoretical Biology 445, $1-8$.

Madadelahi, M., L. F. Acosta-Soto, S. Hosseini,
S.O. Martinez-Chapa and M.J. Madou (2020) Mathematical modeling and computational analysis of centrifugal microfluidic platforms: a review. Lab Chip 20 1318-1357.

Marschewski, J., R. Brechbühler, S. Jung, P. Ruch, B. Michel and D. Poulikakos (2016) Significant heat transfer enhancement in microchannels with herring bone inspired microstructures. International journal of Heat and Mass Transfer 95, 755-764.

Miguel, A. F. (2019) Towards methodologies for optimal fluid networks design. Journal of Applied Fluid Mechanics 12, 1223-1229.

Miguel, A. F. (2010) Natural flow systems: acquiring their constructal morphology. International Journal of Design \& Nature and Ecodynamics 5, 230-241.

Miguel, A. F. (2013) The emergence of design in pedestrian dynamics: locomotion, selforganization, walking paths and constructal law. Physics of Life Reviews 10, 168-190.

Miguel, A. F. (2015) Fluid flow in a porous treeshaped network: optimal design and extension of Hess-Murray's law. Physica A 423, 61-71.

Miguel, A. F. (2016) A study of entropy generation in tree-shaped flow structures. International Journal of Heat and Mass Transfer 92, 349359.

Nguyen, N. T., and Z. Wu (2005) Micromixers-a review. Journal of Micromechanics and Microengineering 15, 1-16.

Omori, T., T. Ishikawa, D. Barthès-Biesel, A.-V. Salsac, Y. Imai, and T. Yamaguchi (2012) Tension of red blood cell membrane in simple shear flow. Physical Review E 86, 056321.

Orsi, G., M. Roudgar, E. Brunazzi, C. Galletti and R. Mauri (2013) Water-ethanol mixing in Tshaped microdevices. Chemical Engineering Science 95, 174-183.

Ritter, P., A. Osorio-Nesme, and A. Delgado (2016) numerical simulations of passive mixing in a microchannel with nozzle-diffuser-like obstacles. International Journal of Heat and Mass Transfer 101, 1075-1085.

Shah, T. R., H. Koten, and H. M. Ali (2020) Hybrid Nanofluids for Convection Heat Transfer. Academic Press, London.

Shaha, I., S. W. Kima, K. Kima, Y. H. Dohb and K. H. Choia (2019) Experimental and numerical analysis of Y-shaped split and recombination micro-mixer with different mixing units. Chemical Engineering Journal 358, 691-706.

Soni, B., A. F. Miguel and A. K. Nayak (2020) A mathematical analysis for constructal design of tree flow networks under unsteady flow. Proceedings of the Royal Society A 476, 20200377.

Tabeling, P. (2005) Introduction to Microfluidics. 
E. Cetkin et al. / JAFM, Vol. 14, No. 5, pp. 1389-1397, 2021.

Oxford University Press, Oxford.

Viktorov, V., R. Mahmud and C. Visconte (2016) Numerical study of fluid mixing at different inlet flow-rate ratios in tear-drop and chain micromixers compared to new $\mathrm{H}-\mathrm{C}$ passive micromixer. Engineering Applications of Computational Fluid Mechanics 10, 182-192.

Wangikar, S. S., P. K. Patowari and R. D. Misra
(2018) Numerical and experimental investigations on the performance of a serpentine microchannel with semicircular obstacles. Microsystem Technologies 24, 3307-3320.

Zhan, X., G. Chen and D. Jing (2020) Optimal analysis of the hydraulic and mixing performances of symmetric T-shaped rectangular microchannel mixer. Fractals. 